Ágora Rev. Cient. 2019; 06(02):e2

\title{
Eficacia de dos esquemas de tratamiento anti-VIH utilizados en el Hospital Militar Central
}

\author{
Efficacy of two anti-HIV treatment schemes at the Central Military Hospital \\ Luis Alberto Sotelo Novoa ${ }^{1}$, Edgar Robert Tapia Manrique ${ }^{1}$, Yovani Martín Condorhuamán ${ }^{1}$
}

\section{RESUMEN}

Objetivo: Determinar la eficacia de dos esquemas de tratamiento anti-VIH utilizados en el Hospital Militar Central durante el periodo de octubre 2017 a mayo 2018. Materiales y Métodos: Este es un estudio observacional, descriptivo, transversal de corte retrospectivo, donde el tamaño de la muestra fueron 52 pacientes con infección por VIH tratados con un esquema inicial antirretroviral en el Hospital Militar Central atendidos durante 6 meses en el período de octubre 2017 a mayo 2018. Resultados: Los resultados demostraron que entre los dos esquemas antiretrovirales Inhibidor Nucleósido de la Transcriptasa Reversa más Inhibidores de Proteasa $(I N T R+I P)$ e Inhibidor Nucleósido de la Transcriptasa Reversa más Inhibidor No Nucleósido de la Transcriptasa Reversa $(I N T R+I N N T R)$, ambos demostraron tener un aumento de recuento de células $\mathrm{CD} 4+$ pero el más eficaz en ser una terapia efectiva fue el esquema antirretroviral $(I N T R+I N N T R)$ obteniendo un recuento final de células CD4+ mayor a 500 células $/ \mathrm{mL}$. Conclusión: Se concluye que el tratamiento (INTR+INNTR) es el más eficaz que el (INTR+IP) en pacientes con infección de VIH en el Hospital Militar Central, siendo la gran mayoría del sexo masculino y con estudios de solo primaria completa con un rango de edad de 29 y los 38 años de edad.

Palabras clave: VIH, antiretrovirales, células CD4+.

\begin{abstract}
Objective: To determine the efficacy of two anti-HIV treatment schemes at the Central Military Hospital during the period from October 2017 to May 2018. Materials and methods: This was an observational, descriptive, cross-sectional and retrospective study, where the sample size was 52 patients. Central Military served during the period from October 2017 to May 2018. Results: The results showed that between the two antiretroviral schemes $(I N T R+I P$ and INTR $+I N N T R)$ both caused an increase in the CD4+ cell count but the most effective therapy was the antiretroviral regimen $(I N T R+I N N T R)$ obtaining a final result of CD4+ cells higher than 500 cells $/ \mathrm{mL}$. Conclusion: It is concluded that the treatment (INTR + INNTR) is more effective than the $(I N T R+I P)$ in patients with HIV infection in the Central Military Hospital, being the great majority of the male sex and with basis studies with a age range of 29 and 38 years old.
\end{abstract}

Keywords: HIV, antiretrovirals, CD4+ cells.

${ }^{1}$ Universidad Nacional Mayor de San Marcos. Facultad de Farmacia y Bioquímica. Lima - Perú.

\section{INTRODUCCIÓN}

El virus de la inmunodeficiencia humana (VIH) a nivel mundial es considerado el problema de salud pública de mayor importancia y uno de los más grandes desafíos para la vida y la dignidad de los seres humanos. Afecta a todas las personas sin distinción de su condición económica, edad, raza, sexo, condición ocupacional, orientación sexual o identidad de género; observándose que los habitantes de los países en desarrollo como el nuestro son los más afectados. (MINSA, 2015).

Con el conocimiento y la experiencia adquirida hasta la actualidad en el tratamiento antirretroviral de gran eficacia (TARGA) y sus beneficios demostrados en la disminución de la morbimortalidad, es pertinente y necesario el realizar estudios que incrementen la información acerca de la respuesta de la infección por VIH a estos tratamientos y más aún, estudiar la respuesta 
del VIH en pacientes nunca antes tratados. (MINSA, 2005).

El tratamiento antirretroviral de gran actividad (TARGA), se viene brindando gratuitamente por el MINSA a toda persona con VIH/SIDA desde hace aproximadamente 12 años; esto incluye a los casos que son reportados en las Fuerzas Armadas y la Policía Nacional del Perú (Bazán-Ruíz, 2013).

En el Ejército Peruano se maneja el tratamiento antirretroviral siguiendo las directrices del Ministerio de Salud (MINSA) que norma al detalle los esquemas a utilizar, la farmacología utilizada consta de la combinación de tres fármacos cuyo mecanismo de acción se basan en la inhibición de la actividad de dos enzimas (transcriptasa reversa y la proteasa) presentes en los linfocitos T CD4+.

Esta supresión enzimática hace posible que la replicación viral disminuya, el recuento de linfocitos T CD4+ aumente y mejore de gran manera la sobrevida de los pacientes.

El presente estudio permitió evaluar la eficacia de los dos esquemas antirretrovirales utilizados en la población infectada con VIH en el Ejército Peruano además de describir las características demográficas.

\section{MATERIALES Y MÉTODOS}

El presente trabajo de investigación se realizó en el Hospital Militar Central con una muestra de 52 pacientes que iniciaron tratamiento antirretroviral VIH durante el período de Octubre 2017 a Mayo 2018. La metodología fue de tipo observacional, descriptivo, transversal con cohorte retrospectivo, usándose la prueba estadística T-Student (Prueba T).

\section{RESULTADOS}

Los resultados obtenidos se presentan de la siguiente manera. En la Fig. 1 no se muestran diferencias significativas a un $95 \%$ de error entre las variables evaluadas: INTR+IP (Tratamiento Antirretroviral que incluye a los Inhibidores de la Proteasa) y INTR+INNTR (Tratamiento Antirretroviral que incluye a un Inhibidor No Nucleósido de Transcriptasa Reversa).

Por otro lado, en la Fig. 2 se indican los tipos tratamientos farmacológicos usados en los pacientes con VIH, siendo el INTR+INNTR el más eficaz. En el grupo de tratamiento antirretroviral INTR+IP se le indicó Indinavir, Zidovudina, Lamivudina y Nelfinavir y en el grupo de tratamiento antirretroviral INTR+INNTR se indicó Efavirenz y Estavudina.

Asimismo, en la Fig. 3 se indican las características de inclusión de los pacientes objeto de estudio. En el 1er. grupo de tratamiento antirretroviral INTR+IP, los pacientes fueron del sexo femenino en un $27 \%$ y del sexo masculino en un $73 \%$. Además, un $39 \%$ se encontraba entre los 39 y 48 años de edad. En el 2do. grupo de tratamiento antirretroviral INTR+INNTR se observó que un $21 \%$ los pacientes eran mayores de 49 años y que en el primer y segundo tratamiento los mayores porcentajes no contaban con estudios superiores.

\begin{tabular}{|l|r|r|}
\hline & INTR + IP & INTR + INNTI \\
\hline Media & $1,960,606,061$ & $2,225,052,632$ \\
\hline Varianza & $2,676,427,371$ & $2,537,661,164$ \\
\hline Observaciones & 33 & 19 \\
\hline Diferencia hipotética de las medias & 0 & \\
Grados de libertad & 39 & \\
Estadístico t & $-0,570765016$ & \\
P(T<=t) una cola & 0,285716626 & \\
Valor crítico de t (una cola) & $1,684,875,122$ & \\
P(T<=t) dos colas & 0,571433253 & \\
Valor crítico de t (dos colas) & $202,269,092$ & \\
\hline
\end{tabular}

Figura 1. Incremento en el número de células CD4+ en los dos esquemas de tratamiento antirretroviral estudiados.

\begin{tabular}{|c|c|c|c|}
\hline \multicolumn{2}{|c|}{ INTR + IP } & \multicolumn{2}{|c|}{ INTR + INNTR } \\
\hline \multicolumn{2}{|c|}{ N (\%) } & \multicolumn{2}{|c|}{ N (\%) } \\
\hline Total & $33(100)$ & Total & $19(100)$ \\
\hline IDV AZT 3TC & $25(75,8)$ & EFV AZT 3TC & $16(84,2)$ \\
\hline NELF AZT 3TC & $8(24,2)$ & EFV D4T 3TC & $3(15,8)$ \\
\hline $\begin{array}{l}\text { ZT: Zidovudina, 3TC: } \\
\text { favirenz, IP: Inhbibidore }\end{array}$ & ivudina, D4T & $\begin{array}{l}\text { IDV: Indinavir, NE } \\
\text { dores no nucleósido }\end{array}$ & $\begin{array}{l}\text { elfinavir, EFV } \\
\text { a transcriptas }\end{array}$ \\
\hline
\end{tabular}

Figura 2. Esquema de tratamientos antirretrovirales utilizados en el presente estudio.

\begin{tabular}{|c|c|c|c|c|}
\hline \multirow[b]{3}{*}{ Características } & \multicolumn{4}{|c|}{ Esquemas } \\
\hline & \multicolumn{2}{|c|}{ INTR + IP } & \multicolumn{2}{|c|}{ INTR + INNTR } \\
\hline & $\mathbf{N}^{\circ}$ & $\%$ & $\mathbf{N}^{\circ}$ & $\%$ \\
\hline \multicolumn{5}{|l|}{ Sexo } \\
\hline Masculino & 24 & 72,7 & 17 & 89,5 \\
\hline Femenino & 9 & 27,3 & 2 & 10,5 \\
\hline \multicolumn{5}{|l|}{ Grupos etarios } \\
\hline $18-28$ & 9 & 27,3 & 5 & 26,3 \\
\hline $29-38$ & 13 & 39,4 & 5 & 26,3 \\
\hline $39-48$ & 10 & 30,3 & 5 & 26,3 \\
\hline$>49$ & 1 & 3 & 4 & 21,1 \\
\hline \multicolumn{5}{|l|}{$\begin{array}{l}\text { Grado de } \\
\text { instrucción }\end{array}$} \\
\hline Primaria & 19 & 57,6 & 6 & 31,6 \\
\hline Secundaria & 7 & 21,2 & 8 & 42,1 \\
\hline Superior & 7 & 21,2 & 5 & 26,3 \\
\hline
\end{tabular}

Figura 3. Características generales de la población en estudio. 
Finalmente, en la Figura 4 indica el incremento del número de células CD4+ según esquema de tratamiento antirretroviral, y en la Figura 5 se indica el incremento en los conteos finales de células CD4+ después de 6 meses de tratamiento.

\begin{tabular}{ccc}
\hline $\begin{array}{c}\text { Incremento } \\
\text { de CD4 } \\
\text { (cel/mL) }\end{array}$ & INTR + IP & INTR + INNTR \\
\hline $0-$ & $18(55,0)$ & N (\%) \\
\hline $201-400$ & $10(30,0)$ & $9(42,0)$ \\
$>401$ & $5(15,0)$ & $2(11,0)$ \\
\hline
\end{tabular}

Figura 4. Incremento del número de células CD4+ según esquema de tratamiento antirretroviral

\begin{tabular}{|c|c|c|}
\hline $\begin{array}{c}\text { Conteo } \\
\text { final de } \\
\text { CD4 (cel/ } \\
\text { mL) }\end{array}$ & $\begin{array}{c}\text { INTR + IP } \\
\text { N (\%) }\end{array}$ & INRT + INNTR \\
\hline$<500$ & $17(51,5)$ & $8(42,1)$ \\
\hline 500 a mas & $16(48,5)$ & $11(57,9)$ \\
\hline TOTAL & $33(100)$ & $19(100)$ \\
\hline
\end{tabular}

Figura 5. Rango de conteo final de células CD4+ después de 6 meses de tratamiento

\section{DISCUSIÓN}

En base a los resultados obtenidos, no se reportó una diferencia estadísticamente significativa en el incremento de células CD4+ de ambos esquemas; en consecuencia, las diferencias que se evidencian entre los valores promedios podrían deberse a otros factores que de alguna manera modifican el efecto del tratamiento; dentro de estos podríamos mencionar a la mala adherencia al tratamiento, farmacología asociada a la comorbilidad o una dosis insuficientes, resistencia a los fármacos antirretrovirales, interacciones medicamentosas, entre otros.

El $78.8 \%$ de la población fue masculina (72.7\% para las observaciones del esquema INTR + IP y $89,5 \%$ para el esquema INTR + INNTR), con un promedio de edad entre 29 a 38 años, con un grado de instrucción primaria.

Ambos esquemas demostraron ser eficaces en el incremento de células CD4+, con un margen a favor del esquema que incluye a un INNTR que fue el grupo que presentó un mayor porcentaje en el incremento de CD4 en el rango 201-400 células $/ \mathrm{mL}$; estos resultados confirman a los obtenidos en estudios similares en la ciudad de
Colombia y en nuestro país (Machado, 2014; Núñez, 2017).

En el recuento final de células CD4+ llevado a cabo a los 6 meses de tratamiento, se evidencia una mejor eficacia del esquema que incluye un INNTR con un $57.9 \%$ frente a los IP con $48.5 \%$ para alcanzar un valor sanguíneo de células CD4+ superiores a 500 células $/ \mathrm{mL}$, valor corte para continuar con la terapia sin modificaciones. Estos resultados refuerzan los encontrados en estudios similares en el Perú y en dos ciudades de Colombia (Machado, 2014; Núñez, 2017).

\section{CONCLUSIONES}

$\mathrm{Se}$ concluye que el tratamiento (INTR+INNTR) es más eficaz que el (INTR+IP) en pacientes con infección de VIH en el Hospital Militar Central, siendo la gran mayoría del sexo masculino y con estudios de solo primaria completa con un rango de edad de 29 y los 38 años de edad.

\section{AUTOR DE CORRESPONDENCIA}

Dr. QF. Edgar Robert Tapia Manrique

Facultad de Farmacia y Bioquímica

Universidad Nacional Mayor de San Marcos

E-mail: edgar_tapia1706@hotmail.com

\section{REFERENCIAS BIBLIOGRÁFICAS}

1. Bazán - Ruíz, S., Chanamé, L. y Maguiña, C. (2013). Adherencia al TARGA en VIH /SIDA. Un Problema de Salud Pública. Acta Médica Peruana, 30(2).

2. Codina, C., Martín, T., Ibarra, O. 2002. Infección por el virus de la Inmunodeficiencia Humana. Fundación Española de Farmacia Hospitalaria FEFH. 3ra Edición.

3. Echevarría, J., López de Castilla, D., Iglesias, D., Seas, C., González, E., Maldonado., et al. (2007). Efecto de la terapia antiretroviral de gran actividad (TARGA) en pacientes enrolados en un Hospital Público en LimaPerú. Revista Médica Herediana, 18(4).

4. Machado-Alba, J., and Vidal, X. (2012). Effectiveness of antiretroviral treatment in Colombia. Revista Panamericana de Salud Pública, 32(5): 360-7.

5. Masiá, M., Padilla, S.; Barber, X., Terol, J., Lidón, F. and Gutiérrez. (2016). Comparative Impact of Suppressive Antiretroviral 
Regimens on the CD4/CD8 T-Cell Ratio. Medicine, 95(11).

6. MINSA. NTS No004-MINSA/DGSP-V.02. 2005. Norma técnica para el tratamiento antirretroviral de gran actividad - TARGA en adultos infectados por el virus de la inmunodeficiencia humana.

7. MINSA. NTS $\mathrm{N}^{\circ} 097$ - MINSA/DGSP-V.02 2014. Norma técnica de salud de atención integral del adulto mayor con infección por el virus de la inmunodeficiencia humana (VIH).

8. MINSA. Boletín Mensual. Setiembre 2018. Centro Nacional de Epidemiología, Prevención y Control de Enfermedades. Situación epidemiológica del VIH-Sida en el Perú.

9. MINSA. Decreto Supremo No 035-2015-SA. 2015. Plan estratégico multisectorial de prevención y control de ITS, VIH y SIDA. PEM 2015 - 2019.

10.Núñez, Z. (2017). Eficacia de dos esquemas iniciales de tratamiento antirretroviral en pacientes con infección por VIH: Hospital Nacional Edgardo Rebagliati Martins, Lima 1999 - 2006. (Tesis de especialidad). Universidad Nacional Mayor de San Marcos, Lima-Perú.

11.OMS: WHO/HIV/2018.05 (2018) Estrategia mundial del sector de la salud contra el VIH, 2016-2021.

12.Ribera, E., Tuset, M., Martín, M y Elena del Cacho. (2011). Características de los fármacos antirretrovirales. Formación médica continuada: Infección por el VIH en el adulto. Revista de Enfermedades Infecciosas y Microbiología Clínica, 29(5):362-391.

13. Staszewski, S., Morales-Ramirez, J., Tashima, K., Rachlis, A., Skiest, D., Stanford, J. et al. (1999). Efavirenz plus zidovudine and lamivudine, efavirenz plus indinavir, and indinavir plus zidovudine and lamivudine in the treatment of HIV-1 infection in adults. The New England Journal of Medicine, 341:18651873.

14. Wiesmann, F., Braun, P., Knickmann, M., Knechten, H. 2014. Low level HIV viremia is more frequent under protease-inhibitor containing firstline therapy than under NNRTI - regimens. 